\title{
ESTUDO DO COMPORTAMENTO FLUIDODINÂMICO DE UM LEITO VIBROFLUIDIZADO PARA DIFERENTES AMPLITUDES E FREQUENNCIAS
}

\author{
A. B. S. COSTA, F. B. FREIRE e J. T. FREIRE
}

\author{
Universidade Federal de São Carlos, Departamento de Engenharia Química, Centro de Secagem e \\ Sistemas Particulados \\ E-mail para contato: freire@ufscar.br
}

\begin{abstract}
RESUMO - O emprego de partículas inertes com diâmetros superiores a 2,0mm, para a secagem de pasta no leito vibrofluidizado, ainda necessita de estudos como a caracterização fluidodinâmica do leito diante das diferentes condições operacionais. Assim o objetivo do presente trabalho consiste em investigar o comportamento fluidodinâmico do leito vibrofluidizado submetido a duas amplitudes (A), 0,015m e $0,003 \mathrm{~m}$, associadas a diferentes frequências (f), que foram quantificadas de acordo com o número adimensional de vibração $(\Gamma)$. Com isso, verificou-se a influência que estas variáveis, exercem na queda de pressão máxima do leito, na velocidade de mínima fluidização e no desvio padrão da queda de pressão, utilizando para isso esferas de vidro com 3,19mm de diâmetro, como material inerte. Os resultados mostraram que o comportamento fluidodinâmico é dependente das combinações entre A e f, e não univocamente do número adimensional de vibração.
\end{abstract}

\section{INTRODUÇÃO}

O leito vibrofluidizado é um equipamento que corresponde a um leito fluidizado comum com a adição de uma agitação mecânica, com movimentos verticais ou horizontais, e tem a finalidade de melhorar a característica do transporte e a qualidade da agitação das partículas no interior do leito. Este tipo de equipamento pode ser aplicado em vários setores e indústrias, podendo ser utilizado na secagem de materiais granulares (Silva-Moris e Costa 2006), na secagem de materiais pastosos (Meili et al. 2010), no recobrimento de partículas (Nunes et al. 2012) e na granulação (Costa et al. 2011).

Para a secagem de pastas com o auxílio do material inerte, existentes vários tipos de equipamentos que podem ser promissores para o desenvolvimento desta operação. No entanto, o leito vibrofluidizado tem apresentado destaque, uma vez que, a vibração desenvolvida neste equipamento em conjunto com o escoamento do fluido, contribui com a agitação do material inerte e facilita a fluidização, principalmente, de matérias com características coesivas e pastosas. Além de reduzir aglomeração de partículas, canais preferenciais e bolhas durante o processo. Outros fatores que favorecem este tipo de leito são as efetivas taxas de transferência de calor e massa, e a redução do consumo de energia, visto que, os valores dos parâmetros, velocidade de mínima fluidização e queda de pressão no interior do leito, são menores quando comparados com leitos fluidizados convencionais 
(Gupta e Mujumdar 1980; Kunii e Levenspil 1991).

Com a finalidade de compreender os mecanismos e fenômenos envolvidos durante a secagem de pasta no leito vibrofluidizado, é importante caracterizar o comportamento dinâmico das partículas diante das variáveis operacionais envolvidas no processo e das características físicas do particulado inerte. Daleffe et al. (2008), mostraram que as curvas fluidodinâmicas são influenciadas pela característica do leito de partículas e também pelo efeito da ação vibracional aplicada.

A intensidade com que a vibração é produzida neste equipamento pode ser quantificada de diversas maneiras, porém, muitos trabalhos baseiam-se principalmente no número adimensional de vibração $(\Gamma)$, de acordo com a definição apresentada na Equação (1).

$\Gamma=\frac{A(2 . \pi \cdot f)^{2}}{g}$

onde A é a amplitude, f a frequência vibracional e $\mathrm{g}$ a aceleração gravitacional.

$\mathrm{Na}$ literatura encontram-se diversos trabalhos sobre o comportamento fluidodinâmico gáspartícula no leito vibrofluidizado. Entretanto alguns autores, como Daleffe, et al. (2005), observaram a não universalidade do adimensional de vibração, uma vez que $\Gamma$ de mesmo valor resultou em comportamentos fluidodinâmicos distintos. Meili et al. (2010) comprovaram que o $\Gamma$ não pode ser usado unicamente para caracterizar a dinâmica do leito, destacando a importância de fornecê-lo juntamente com A ou com f para demonstrar o efeito da vibração. No entanto, Nunes et al. (2012), obtiveram um comportamento interessante nas curvas fluidodinâmicas, em que a mesma amplitude combinadas a duas frequências diferentes resultou em valores muito próximo da queda de pressão.

Com a intuito de dar continuidade aos estudos sobre o comportamento dinâmico do leito vibrofluidizado, neste trabalho selecionou-se dois valores de amplitudes, um baixo de 0,003m e outro alto de $0,015 \mathrm{~m}$, combinadas a frequências que variaram de $100 \mathrm{em} 100 \mathrm{rpm}$. O objetivo consiste em realizar uma investigação mais detalhada da influência que a amplitude, a frequência de vibração e o número adimensional de vibração, podem exercer nos parâmetros velocidade de mínima fluidização $\left(\mathrm{U}_{\mathrm{mf}}\right)$, queda de pressão $(\Delta \mathrm{p})$ e desvio padrão de queda de pressão $(\delta \mathrm{p})$, na presença de um material inerte com diâmetro de $3,19 \mathrm{~mm}$.

\section{MATERIAIS E MÉTODOS}

\subsection{Material e Equipamento}

A unidade experimental utilizada para o desenvolvimento deste trabalho, pode ser encontrado nos trabalhos de Meili et al. (2010) e Meili et al. (2012). Como inerte utilizou-se esferas de vibro com massa específica de $2500 \mathrm{~kg} / \mathrm{m}^{3}$ e diâmetro médio de Sauter de 3,19mm. Para o desenvolvimento dos ensaios, a altura do leito estático foi de $9 \mathrm{~cm}$, equivalente a uma massa total de $1,5 \mathrm{~kg}$ de inertes. 


\subsection{Procedimento Experimental}

A princípio foram efetuados alguns testes, a fim de determinar quais os intervalos de frequência que seriam selecionadas para a realização dos ensaios experimentais, uma vez que, preferiu-se manter as duas amplitudes trabalhadas por Meili et al. (2012). Estas amplitudes correspondem às condições máxima $(0,015 \mathrm{~m})$ e mínima $(0,003 \mathrm{~m})$ de operação estável do leito projetado. Depois de selecionado o intervalo da frequência vibracional a ser trabalhada e determinado o adimensional de vibração de cada combinação de A e f, as condições vibracionais estão organizadas na Tabela 1.

TABELA 1 - Condições experimentais

\begin{tabular}{cccc}
\hline $\begin{array}{c}\text { Condições } \\
\text { experimentais }\end{array}$ & $\begin{array}{c}\text { Amplitude } \\
(\mathrm{m})\end{array}$ & $\begin{array}{c}\text { Frequência } \\
(\mathrm{rpm})\end{array}$ & $\Gamma$ \\
\hline 1 & 0,003 & 400 & 0,53 \\
2 & 0,003 & 500 & 0,84 \\
3 & 0,003 & 600 & 1,2 \\
4 & 0,003 & 700 & 1,65 \\
5 & 0,003 & 800 & 2,15 \\
6 & 0,003 & 900 & 2,7 \\
7 & 0,003 & 1000 & 3,36 \\
8 & 0,003 & 1100 & 4,06 \\
9 & 0,015 & 400 & 2,69 \\
10 & 0,015 & 500 & 4,19 \\
11 & 0,015 & 600 & 6,04 \\
\hline
\end{tabular}

Os ensaios foram realizados a temperatura de $30^{\circ} \mathrm{C}$ e para obtenção das curvas fluidodinâmica, primeiramente, os parâmetros eram ajustados conforme apresentado na Tabela 1. Em seguida, o leito era mantido fluidizando até que fosse alcançado o regime permanente. Com o leito estabilizado, iniciava-se a obtenção dos dados de queda de pressão em função da redução da velocidade superficial do ar (Us), metodologia clássica vista em Bratu e Jinescu (1971). Paralelamente, o sistema de aquisição de dados fornecia os valores do desvio padrão da queda de pressão, por meio de 1024 pontos obtidos em intervalos de três segundo, para auxiliar nas análises fluidodinâmicas, conforme proposto por Garim (1998).

A determinação da velocidade de mínima fluidização no leito vibrofluidizado foi realizada conforme a metodologia empregada em leitos fluidizados convencionais, correspondendo a inserção de duas retas tangentes a curva de queda de pressão em função da velocidade superficial do ar, uma tangente na região de leito fixo e outra tangente na de leito fluidizado (Kunii e Levenspil 1991). Com a finalidade de assegura a confiabilidade dos dados, realizou a réplica dos dados para o leito fluidizado e escolheu-se duas condições aleatórias do vibrofludizado, uma referente a $A=0,003 \mathrm{~m}$ e uma a $A=0,015 \mathrm{~m}$, e realizou a reprodução destes ensaios para cinco repetições. 


\section{RESULTADOS E DISCUSSÕES}

Na Figura 1 estão apresentados as curvas da queda de pressão e os desvios padrões da queda de pressão em função da velocidade superficial do ar, para os dados obtidos no leito fluidizado convencional e para as duas amplitudes trabalhadas no leito vibrofluidizado.
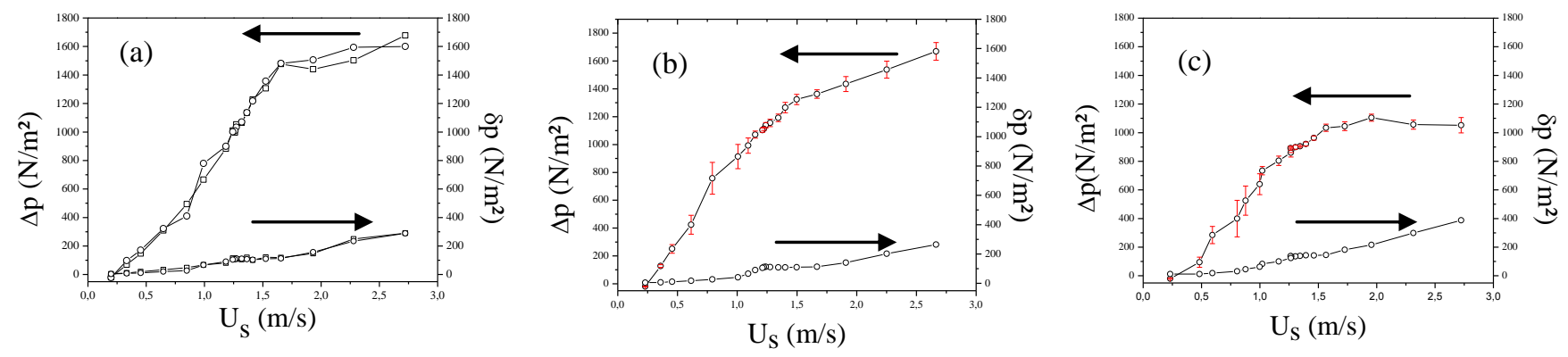

Figura 1- Queda de pressão $(\Delta \mathrm{p})$ do leito e desvio padrão da queda $(\delta \mathrm{p})$ de pressão em função da $\mathrm{U}_{\mathrm{S}}$, para (a) o leito fluidizado e (b) o leito vibrofluidizado com $A=0,003 \mathrm{~m}$ e $800 \mathrm{rpm}(\Gamma=2,15)$ e (c) o leito vibrofluidizado com $A=0,015 \mathrm{~m}$ e $500 \mathrm{rpm}(\Gamma=4,12)$.

Na Figura 1-a, os dados exibiram um comportamento característico de um leito fluidizado, onde é evidente a transição bem definida entre o leito fixo e leito fluidizado, no instante que a velocidade superficial do ar é 1,6m/s, mesmo para partículas com 3,19mm de diâmetro. As curvas indicam também uma boa reprodutibilidade de dados, uma vez que, a diferença encontrada entre os dados medidos e a sua réplica foram inferiores a 10\%. No instante que a agitação foi adicionada ao sistema, observa-se nas Figuras 1-b e 1-c, que a região de transição do leito fixo para o leito fluidizado apresentou uma amortização, não ficando evidente, uma velocidade de mínima fluidização para este conjunto de partículas. Com relação à reprodução dos dados, observou-se que independente da intensidade da agitação, os dados exibiram uma boa reprodutibilidade, obtida tanto para os ensaios realizados com $A=0,003 \mathrm{~m}$ quanto para a $A=0,015 \mathrm{~m}$. No entanto, para as duas condições, os gráficos mostraram que os maiores desvios entre os dados encontram-se na região de leito fixo. A dificuldade em reproduzir esta região do leito, está relacionada com a dificuldade de obter o mesmo arranjo das partículas e também devido às tensões existentes, que acabam sendo minimizados após a expansão dos leitos.

A boa reprodutibilidade adquirida pelas curvas fluidodinâmicas, tanto para o leito fluidizado como para o leito vibrofluidizado, assegura a qualidade dos dados diante deste tipo de partícula, visto que, foram utilizadas partículas que correspondem ao grupo D da classificação de Geldart (1973), ou seja, partículas que apresentam durante a fluidização o surgimento de canais preferenciais e bolhas.

A Figura 2 apresenta as curvas da queda de pressão e os desvios padrões da queda de pressão em função da velocidade superficial do ar, no leito vibrofluidizado, para: (a) a amplitude de 0,003m parametrizada nas diferentes frequências, resultando assim, na variação no número adimensional de 

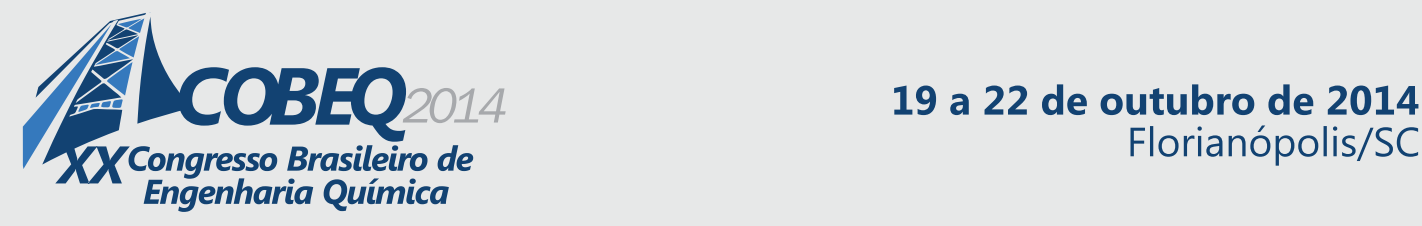

Florianópolis/SC

vibração de 0,53 a 4,06; e (b) a amplitude de 0,015m parametrizada em três frequências, promovendo a variação do $\Gamma$ de 2,69 a 6,04 .

Os resultados encontrados na Figura 2-a, referente à amplitude de 0,003m, demonstraram que a vibração do leito influenciou mais expressivamente na região de leito fixo do que na região de leito fluidizado, confirmando assim o que era esperado, visto que, para esta amplitude o arranjo das partículas determina o comportamento fluidodinâmico. Na região de leito fixo observou-se uma redução na queda de pressão, à medida que, a frequência de vibração foi elevada no equipamento. Para a região de leito fluidizado, os valores da queda de pressão máxima foram muito próximos, para todas as situações analisadas, mostrando a pouca influência deste parâmetro nesta região. Ao verificar a velocidade de mínima fluidização obtidos nos ensaios, observou-se um comportamento esperado, dado que, este parâmetro apresentou um aumento, pouco expressivo, á medida que aumentava o adimensional de vibração (com o aumento da frequência), promovendo uma variação de $1,2 \mathrm{~m} / \mathrm{s}$ para $\Gamma=0,53$ a $1,42 \mathrm{~m} / \mathrm{s}$ para $\Gamma=4,06$.
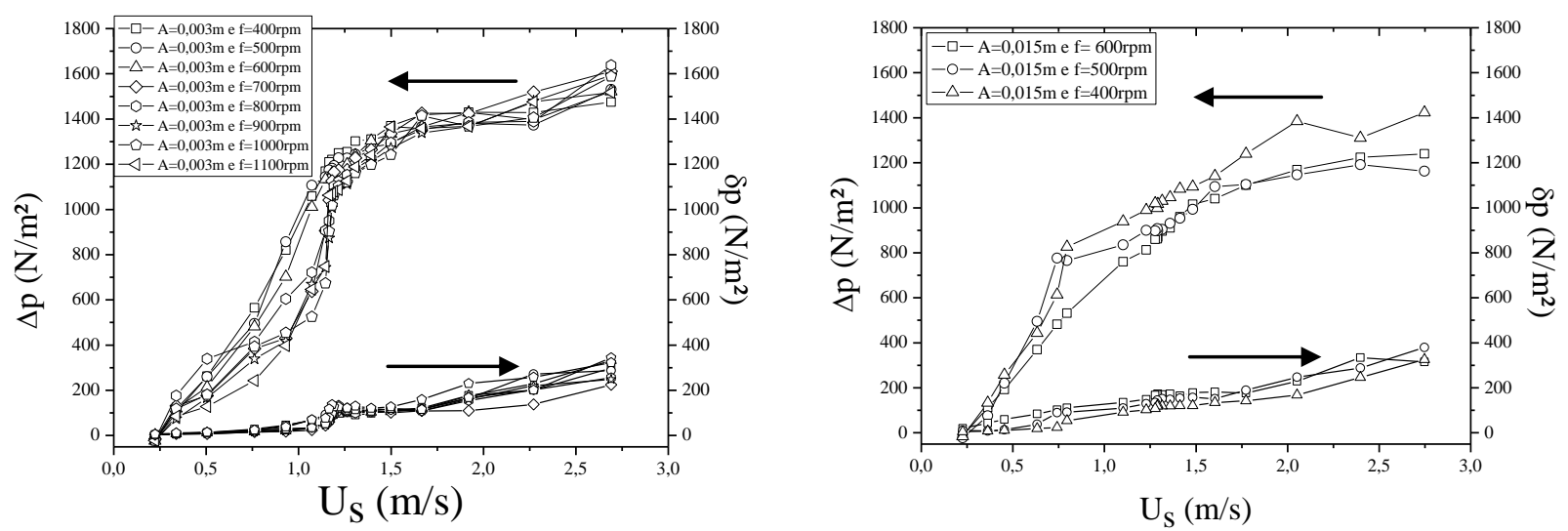

Figura 2- Queda de pressão no leito e o desvio padrão da queda de pressão em função de $U_{\mathrm{s}}$ para o leito vibrofluidizado com (a) $A=0,003 \mathrm{~m}$ e (b) $A=0,015 \mathrm{~m}$, os dois parametrizados na frequência.

A tendência encontrada na região de leito fixo está relacionada, principalmente, com a porosidade e a tortuosidade do meio, pois o aumento da frequência vibracional promoveu o aumento da porosidade, causando assim a redução da queda de pressão. Para a região do leito fluidizado, a porosidade alcançada no material particulado foi praticamente a mesmas em todas as condições, por isso a pouca variação na queda de pressão máxima. Daleffe (2005) ao estudar o comportamento fluidodinâmico de partículas com $2,19 \mathrm{~mm}$ de diâmetro, utilizando a mesma amplitude e variando a frequência, verificou que não foi possível encontrar uma tendência para caracterizar o leito. Visto que, a queda de pressão diminuiu com o aumento da frequência até obter um valor de $\Gamma=2$. Para o $\Gamma=4$, a queda de pressão aumentou. Ao comparar este resultado com os obtidos neste trabalho, mesmo se tratando de partículas com diâmetros maiores, a única tendência encontrada foi na região de leito fixo. $\mathrm{Na}$ região de leito fluidizado pode-se considerar que também não foi encontrado tendência, pois os valores de queda de pressão estão muito próximos, praticamente dentro do erro experimental esperado. 


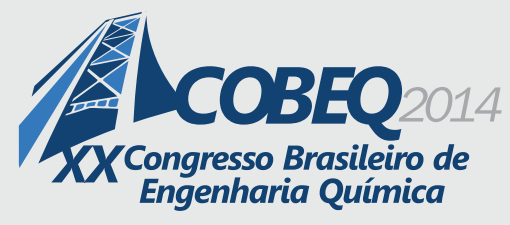

19 a 22 de outubro de 2014

Florianópolis/SC

Ao observar as curvas de desvio padrão da queda de pressão, na região de leito fixo, constatouse que a queda de pressão no leito não apresentou oscilações intensas e se manteve praticamente constante. Este resultado é obtido quando a estrutura interna do conjunto de partículas é mantida sem muitas alterações, fazendo com que a permeabilidade do mesmo fique praticamente constante, não ocorrendo variações relevantes na queda de pressão. No instante que o leito alcançou o estado de fluidização verificou-se que o desvio padrão da queda de pressão sofreu um aumentou, à medida que a velocidade superficial do ar era elevada. Este resultado mostra que se iniciou um movimento mais intenso das partículas, ocasionando assim oscilações na pressão e em consequência, o aumento do desvio padrão. Observou-se também que para todas as situações o comportamento foi o mesmo, além das curvas terem ficado muito próximas umas das outras.

$\mathrm{Na}$ Figura 2-b, que corresponde a maior amplitude utilizada $(0,015 \mathrm{~m})$, observa-se que a dinâmica do leito foi expressivamente mais influenciada. Nesta situação nota-se que o aumento da frequência de vibração de 400 para $500 \mathrm{rpm}$, promoveu uma redução da queda de pressão no interior do leito. Entretanto ao aumentar a frequência de vibração de 500 para $600 \mathrm{rpm}$ a queda de pressão também reduziu, mas apresentou valores muito próximos. Com relação à velocidade de mínima fluidização, observou-se que está variável não apresentou uma considerável alteração com o aumento da frequência de vibração, uma vez que, foram obtidos valores entre 1,4 a 1,55m/s.

Baseados nestes resultados, foi possível constatar que a dinâmica das partículas no interior do leito, para esta amplitude, apresentou uma expansão nítida, facilitando a passagem de ar no interior do leito e reduzindo assim a queda de pressão, com o aumento da frequência vibracional. Daleffe (2005) ao aumentar a amplitude trabalhada, observou a redução da queda de pressão à medida que a frequência vibracional era elevada. Meili et al. (2012), mesmo que tenham trabalhado com partículas menores que $1 \mathrm{~mm}$, observaram comportamento similar para as maiores amplitudes, a medida que foi aumentado a frequência vibracional.

Os resultados encontrados para as curvas do desvio padrão da queda de pressão mostraram que, à medida que a frequência vibracional foi elevada, houve um aumento nos desvios padrões da queda de pressão, com o aumento da velocidade superficial do ar. Este fenômeno ocorreu, pois, esta amplitude produziu uma agitação no material particulado mais intenso e oscilante, o que resultou na instabilidade da pressão no interior do leito, aumentando assim o desvio padrão da queda de pressão à medida que a frequência foi aumentada.

Com o intuito de realizar uma análise mais detalhada do parâmetro vibracional, serão apresentados os resultados dos ensaios em que $\Gamma$ foi mantido constante, variando-se a amplitude e a frequência vibracional. A Figura 3 corresponde à queda de pressão e o desvio padrão da queda de pressão em função da velocidade superficial do ar, para o leito fluidizado e leito vibrofluidizado com o $\Gamma=4,03$, parametrizado na amplitude do leito $A=0$ (referindo-se ao leito fluidizado), $A=0,003 \mathrm{~m}$ e $\mathrm{A}=0,015 \mathrm{~m}$.

Diante destes resultados observa-se que o leito fluidizado apresenta a maior queda de pressão no interior do leito, com um valor de $1600 \mathrm{~N} / \mathrm{m}^{2}$, e uma velocidade de mínima fluidização igual á $1,6 \mathrm{~m} / \mathrm{s}$. Com a adição da agitação ao sistema, observou-se que para um mesmo valor de $\Gamma$ o comportamento 
fluidodinâmico dependeu do par de valores de A e f. Com relação aos dados do leito fluidizado, verifica-se, uma redução na queda de pressão de $13 \%$ para $A=0,003 \mathrm{~m}$ e $23 \%$ para $A=0,015 \mathrm{~m}$. $A U_{\mathrm{mf}}$ do vibrofluidizado também diminui em relação ao leito fluidizado comum, $19 \%$ para $\mathrm{A}=0,015 \mathrm{~m}$ e $12,5 \%$ para $A=0,003 \mathrm{~m}$. Mostrando assim que variação expressiva dos parâmetros esta relacionada com a amplitude (ou frequência) da agitação. Pois na amplitude de 0,015m, as partículas apresentam um deslocamento maior no interior da câmara de secagem, com isso, aumentou-se a porosidade do leito, promovendo a redução da queda de pressão. Porém, o que pode explicar a queda de pressão para $\mathrm{A}=0,003 \mathrm{~m}$ ser superior a $\mathrm{A}=0,015 \mathrm{~m}$ é a reorganização das partículas durante a agitação do sistema. Uma vez que, o leito com $\mathrm{A}=0,003 \mathrm{~m}$ tende a se compactar e, à medida que as partículas vão se rearranjando, vai obstruindo a passagem do ar, obrigando que o mesmo exerça uma pressão maior para percolar o leito do material particulado.

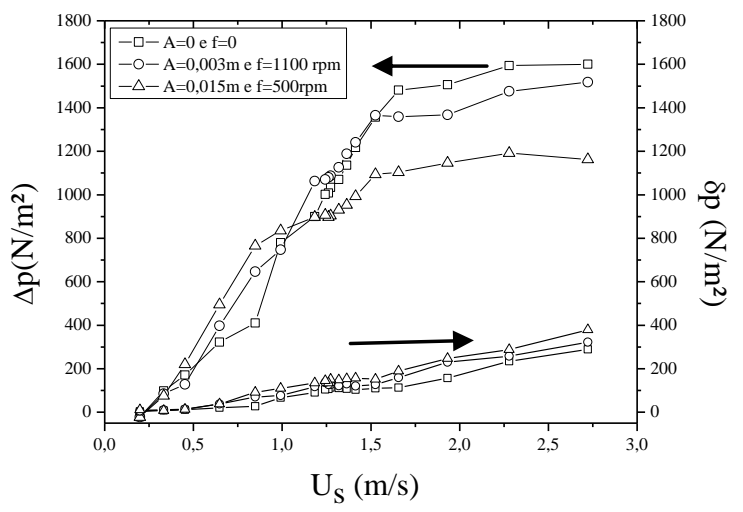

Figura 3- Queda de pressão no leito e o desvio padrão da queda de pressão em função de $U_{s}$, para o leito fluidizado, e vibrofluidizado com $\Gamma=4,03(\mathrm{~A}=0,003 \mathrm{~m}$ e $\mathrm{f}=1100 \mathrm{rpm})$ e $\Gamma=4,19$

$$
(\mathrm{A}=0,015 \mathrm{~m} \text { e } \mathrm{f}=500 \mathrm{rpm})
$$

Ao analisar os resultados das curvas do desvio padrão da queda de pressão para o leito fluidizado e vibrofluidizao, ficou evidente que as curvas são bastante próximas, não houve um diferença significativa em fluidização e vibrofluidização, tanto para amplitude a de $0,015 \mathrm{~m}$ como para $0,003 \mathrm{~m}$.

\section{CONCLUSÃO}

Diante da análise da dinâmica do leito vibrofluidizado, foi observado o comportamento clássico da curva de queda de pressão em função da velocidade superficial do ar, em que há o amortecimento da região de transição do leito fixo para o leito fluidizado. Os resultados indicaram também que, para este tipo de partícula, o aumento da frequência vibracional (juntamente com os aumentos de $\Gamma$ ) não apresentaram consideráveis variações para a amplitude de $0,003 \mathrm{~m}$, porém a velocidade de mínima fluidização sofreu um pequeno aumento, juntamente com a variável f. Com os resultados para a 
amplitude de $0,015 \mathrm{~m}$, notou-se que queda de pressão do leito sofreu uma redução mais expressiva, com o aumento da frequência de vibração, no entanto, a velocidade de mínima fluidização não foi tão influenciada com o aumento de $\mathrm{f}$. Ao analisar o $\Gamma$ de mesmo valor, pode-se concluir que $A$ ou $f$ devem ser apresentados junto a este parâmetro vibracional para a efetiva característica do estado de vibrofluidizado.

\section{REFERENCIAS}

BRATU, E.; JINESCU, G.I. Effect of Vertical Vibrations on the Pressure Drop in a Fluidized Layer. Brit. Chem. Eng., v.16, n.8, p.691-695,1971.

COSTA, S. S.; MORIS, V.A.S.; ROCHA, S.C.S. Influence of process variables on granulation of microcrystalline cellulose in vibrofluidized bed. Powder Technol., v. 207, p. 454-460, 2011.

DALEFFE, R. V. Contribuições para a análise do comportamento fluidodinâmico de um leito vibrofluidizado. Universidade Federal de São Carlos, São Carlos, 2005.

DALEFFE, R. V.; FERREIRA, M.C.; FREIRE, J.T. Effects of binary particle size Distribution on the fluid dynamic behavior of fluidized, vibrated and vibrofluidized beds. Braz. J. Chem. Eng., v.25, n.1, p. 83-94, 2008.

GARIM, M. M., Estudos dos coeficientes de transferência de massa em um leito fluidizado e vibrofluidizado. Universidade Federal de São Carlos, São Carlos 1998.

GELDART, D. Types of Gas Fluidization. Powder Technol.,v. 7,p. 285-292, 1973.

GUPTA, R.; MUJUMDAR, A. S. Aerodynamics of a Vibrated Fluid Bed. The Canad. J. of Chem. Eng., v. 58, p. 332-338, 1980.

KUNII, D.; LEVENSPIEL, O. Fluidization Engineering, 2nd ed., Butterworth-Heinemann,Waltham 1991.

MEILI, L.; DALEFFE, R.V.; FERREIRA, M. C. FREIRE, J.T. Analysis of the Influence of Dimensionless VibrationNumber on the Drying of Pastes in Vibrofluidized Beds. Drying Technol., v. 28, p.402-411, 2010.

MEILI, L.; DALEFFE, R.V.; FREIRE, J.T. Fluid Dynamics of Fluidized and Vibrofluidized Bed Operating with Geldart C Particles. Chem. Eng. Technol., v. 35, n. 9, p. 1649-1656, 2012.

NUNES, J.F.; ALCANTARA, F.C.A.; SILVA-MORIS, V.A.S.; ROCHA, S.C.S. Fluid dynamics and coating of sodium bicarbonate in a vibrofluidized bed. Chem. Eng. and Process., v. 52, p. 34-40. 2012.

SILVA-MORIS, V.A.; ROCHA, S.C.S. Vibrofluidized Bed Drying of Adipic Acid. Drying Technol. v. 24, p. 303-313, 2006. 